\title{
Significance tests and their interpretation: An example utilizing published research and $\omega 2$
}

\author{
JAMES R. CRAIG, CHARLES L. EISON, and LEROY P. METZE \\ Western Kentucky University, Bowling Green, Kentucky 42101
}

\begin{abstract}
The issue of the interpretation of significance tests is addressed. An arugment is presented that some measure of association such as $\omega^{2}$ should be provided as an interpretation/decision-making aid for scientific consumers and journal editors. Published research articles were examined regarding use of measures of association and the relationship between sample size and the amount of variance shared by the independent and the dependent variable. The results indicated that no articles reported measures of association and that many published studies are based upon small degrees of relationship between the independent and dependent variables. A change in report-writing and journal-editing practices is suggested.
\end{abstract}

Behavioral scientists have discussed at length what the concept of statistical significance means and how it should be interpreted in the decision-making process associated with tests of significance (e.g., Bakan, 1966; Dwyer, 1974; Gaito \& Firth, 1973; Levine, 1974; Minturn, 1971; Skipper, Guether, \& Nass, 1967). The problem is often discussed in statistics textbooks and has been succinctly stated by Hays (1973): "The rejection of the hypothesis of no difference between population means is tantamount to the assertion that the treatment given does have some statistical association with the dependent variable score.

"However, the occurrence of a significant result says nothing at all about the strength of the association between treatment and score. A significant result leads to the inference that some association exists, but in no sense does this mean that an important degree of association necessarily exists. Conversely, evidence of a strong statistical association can occur in data even when the results are not significant. The game of inferring the true degree of statistical association has a joker: this is the sample size (p. 413)."

The point is that the evaluation of the value or meaningfulness of a statistical outcome is often not based solely upon the probability associated with the test (i.e., the level of significance) but upon those outcomes that are of the most interest for reasons of theory, usefulness, and/or societal implications. Furthermore, the probability associated with a test of significance is greatly influenced by sample size, when other factors are held constant, and does not reflect the degree of relationship between the independent and dependent variables. That is, given a large enough sample size, a significant result may be identified when there is very little association between

Requests for reprints should be sent to: James R. Craig, Department of Psychology, Western Kentucky University, Bowling Green, Kentucky 42101. J. M. Bloom sponsors this paper and takes full editorial responsibility for its content. the independent and dependent variables. Because of this fact, Hays as well as others (e.g., Bolles \& Messick, 1958; Friedman, 1968) have argued that some index of association be utilized to judge the strength of the relationship whether the test of significance is Student's $t$, analysis of variance, chi-square, or some other statistic.

Hays (1973) has presented an index of associative strength for Student's $t$ where the quantity $\omega^{2}$ (Greek omega, squared) is defined as follows:

$$
\omega^{2}=\frac{t^{2}-1}{t^{2}+\left(N_{1}+N_{2}-1\right)},
$$

where $\mathrm{t}^{2}=$ the squared value of the obtained Student $t$ ratio, $N_{1}=$ the number of observation in the first sample, and $\mathrm{N}_{2}=$ the number of observations in the second sample (Hays, 1973, p. 417). The quantity $\omega^{2}$ can be viewed as the proportion of the variance shared by the independent and dependent variables and, as such, it is virtually identical to the squared correlation coefficient. Thus, the concept is not new to behavioral scientists nor should it be subject to gross misinterpretation. Furthermore, the calculation of $\omega^{2}$ takes into account sample size because, as sample size increases while holding other things constant, it takes a progressively smaller difference between the means of two samples to be judged significant via a test of significance such as Student's t. That is, as sample size increases, the minimum degree of association (i.e., the amount of shared variation) required to conclude significance decreases. An example follows.

A recent study (Flagg \& Medin, 1973) utilized two groups of seven subjects each and reported a significant mean difference between the groups with respect to the dependent variable of interest, $\mathrm{t}(12)=3.58, \mathrm{p}<.01$. The associated $\omega^{2}$ value is equal to: 


$$
\omega^{2}=\frac{3.58-1}{3.58+7+7+1}=.458
$$

The $\omega^{2}$ value of .458 thus indicates that approximately $46 \%$ of the variance in the dependent variable is shared with the independent variable. By way of contrast, Gelfand, Hartman, Walder, and Page (1973) reported a significant Student's t statistic for a two-groups comparison involving a total of 136 subjects, $\mathrm{t}(134)=3.10, \mathrm{p}<.01$. The $\omega^{2}$ value would be:

$$
\omega^{2}=\frac{3.10-1}{3.10^{2}+68+68-1}=.063 .
$$

In this instance, the $\omega^{2}$ value of .063 indicates that roughly $6 \%$ of the variance of the dependent variable is shared with the independent variable. Therefore, even though both studies report significant findings, the $\omega^{2}$ index reflects that the amount of variance for which the researchers can account is immensely different in the two studies. The large sample employed in the Gelfand et al. study greatly increased the sensitivity of the Student's t statistic to mean differences thus enhancing the probability of a small amount of shared variance attaining significance. The point is that the value of a significant finding cannot be made solely on the basis of the level of significance attained or the degree of association between independent variables and dependent variables for that matter. Other factors such as the type of dependent variable employed, the research methodology, theoretical relevance, and so on, help determine the value of any statistical outcome. Being able to account for $6 \%$ of the variance in the dependent variable may be deemed quite valuable in one circumstance while being able to account for $46 \%$ of the variance may not be good enough in another situation.

After examining several articles, our interest had been sufficiently piqued to conduct a systematic observation addressing two questions: (1) Do researchers publishing in psychological journals employ measures of association (such as $\omega^{2}$ ) as aids to the interpretation of the significant findings being reported? (2) How large must the shared variance between independent and dependent variables be in order to be published as significant?

\section{METHOD}

For the year 1973, all issues of the Journal of Educational Psychology were examined with six issues being arbitrarily selected for both the Journal of Comparative and Physiological Psychology and the Journal of Personality and Social Psychology. Only those articles which employed Student's $t$ as an analysis technique were utilized, and the following information was gleaned from each article: (1) the first significant Student's t statistic, (2) the sample size, (3) the significance level, and (4) any index of association computed for the Student's $\mathrm{t}$ statistic. If no association index was reported, $\omega^{2}$ was computed for the Student's t statistic. A total of 62 articles from the journal issues selected reported a Student's $t$ statistic. However, 11 were discarded due to insufficient data necessary to compute $\omega^{2}$. Therefore, 51 studies served as the data base.

\section{RESULTS}

Only one researcher reported analyzing the data in a fashion which attempted to assess the magnitude of shared variance between the independent and dependent variable. The other 50 studies reported no measures of association and employed the notion of significant differences as the primary explanatory tool. Rather, the probability associated with any particular statistic was utilized as an index of the "significance" (e.g., the value or meaningfulness) of the findings. For example, one article reported that "the difference was highly significant" $(p<.001)$ and subsequently implied the finding was more "valuable/meaningful" than another result where the "value was significantly different" $(p<.05)$.

The size of $\omega^{2}$ for each of the 51 studies was plotted against sample size and is shown in Figure 1. Examination of the figure shows that there is a great deal of variation among reported significant statistical outcomes in terms of the amount of shared variation present between the independent and dependent variables with some large sample studies being able to explain more variation than some of the studies based upon small samples. However, general studies which employed large samples tended to have a small amount of shared variance with the opposite being observed with smaller samples. Thus, merely calling a result significant and proceeding to discuss implications, to build and/or criticize theory, and to suggest future research ignores the fact that often only

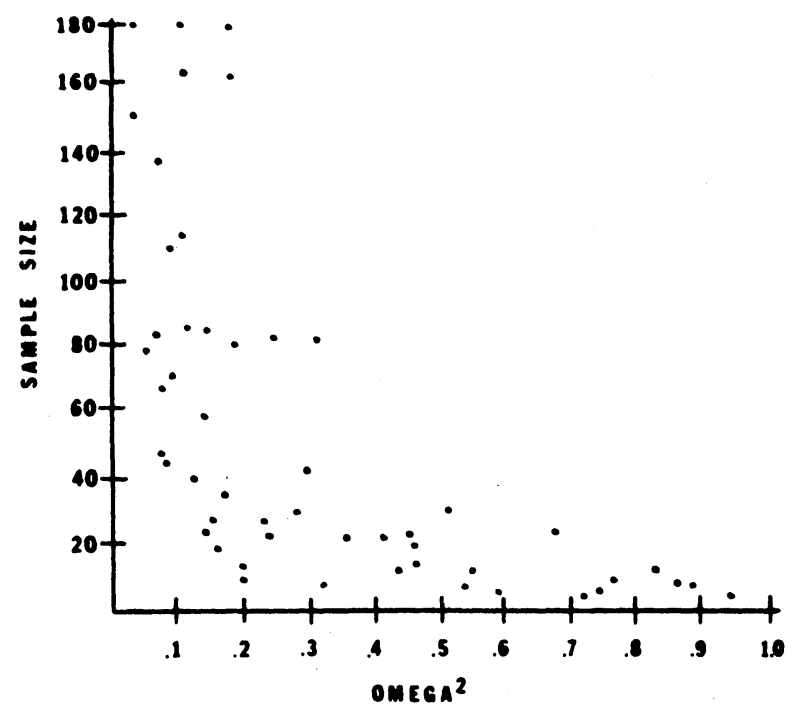

Figure 1. Scatter diagram of 51 different studies comparing sample size and the computed $\omega^{2}$ value where $t$ values were of various levels of significance. 
a small portion of the variation of the dependent variable is "explainable."

These data would also imply that researchers and journal editors as a whole tend to (over)rely on "significant" differences as the definition of meaningful research. That is, the significant difference concept is too commonly viewed an end product of the analysis rather than being a first step in trying to explain concomitant variation in the observed data. However, since psychology's task is not one of identifying "significant" differences but "meaningful" relationships among variables, it would appear that dealing only with significant differences is not enough. We believe, therefore, that researchers should be utilizing some index of association as an additional descriptive/analytical tool for understanding research data (whatever index is used is unimportant if it is appropriate to the design and the analysis conducted). One of the arguments some might advance against reporting measures of association is that their computation is cumbersome and / or unwieldy. For $\omega^{2}$, that argument hardly seems justified. but for those lazy ones among us, Table 1 presents the $\omega^{2}$ values for associated tabled ts. To use the table, merely locate the usually accepted significance levels across the top and read down the column to the row that has the appropriate number of degrees of freedom. If the $t$ value for that combination of significance level and degrees of freedom is deemed significant, the $\omega^{2}$ table will indicate the minimal amount of association present. For example, if the computed $t$ value with $15 \mathrm{df}$ had a probability of less than .01 , but greater than .005 , then Table 1 indicates that the amount of shared variance would be at least .2534 .

As Levine (1974) argues, the task of investigator is to present as much relevant evidence as possible to support his case for the identification and elaboration of meaningful relationships. However, part of the burden of changing researchers' habits of reporting and interpreting data lies with journal editors. Besides using indices of association as another tool to use in sorting manuscripts, editors would also help lessen the information explosion by reducing the numbers of studies reporting "weak" results while increasing the overall strength of the discipline. It is not being argued here that indices of association or shared variance be the only criterion utilized for article selection by editors or for determining the value of a study by the behavioral scientist "consumer"-that would only be replacing one arbitrary criterion for another. Nor is it being suggested that researchers use cnly "small" samples so that when a significant outcome is found, the association index will be relatively large. Rather, we are proposing that indices of association are another part of a composite picture a researcher is building when he reports data suggesting one or more variables are important in understanding a particular behavior.
Table 1

The $\omega^{2}$ Values for Commonly Used Levels of Significance for Student's $t^{*}$

\begin{tabular}{|c|c|c|c|c|}
\hline \multicolumn{5}{|c|}{ Levels of Significance } \\
\hline df & .050 & .025 & .010 & .005 \\
\hline 1 & .9283 & .9816 & .9970 & 9992 \\
\hline 2 & .6530 & .8141 & .9224 & .9606 \\
\hline 3 & .4757 & .6460 & .7969 & .8688 \\
\hline 4 & .3714 & .5278 & .6849 & .7710 \\
\hline 5 & .3042 & .4449 & .5959 & .6855 \\
\hline 6 & .2576 & .3840 & .5260 & .6143 \\
\hline 7 & .2235 & .3379 & .4702 & .5554 \\
\hline 8 & .1974 & .3016 & .4249 & .5063 \\
\hline 9 & .1766 & .2723 & .3875 & .4651 \\
\hline 10 & .1599 & .2483 & .3562 & .4297 \\
\hline 11 & .1462 & 2282 & .3295 & .3995 \\
\hline 12 & .1345 & .2112 & .3065 & .3731 \\
\hline 13 & .1247 & .1964 & .2865 & .3499 \\
\hline 14 & .1161 & .1837 & .2689 & .3295 \\
\hline 15 & .1087 & .1724 & .2534 & .3113 \\
\hline 16 & .1022 & .1626 & .2396 & .2950 \\
\hline 17 & .0964 & .1538 & .2273 & .2790 \\
\hline 18 & .0912 & .1458 & .2161 & .2669 \\
\hline 19 & .0865 & .1387 & .2059 & .2549 \\
\hline 20 & .0824 & .1322 & .1968 & .2438 \\
\hline 21 & .0786 & .1264 & .1884 & .2337 \\
\hline 22 & .0751 & .1209 & .1806 & .2245 \\
\hline 23 & .0719 & .1160 & .1736 & .2158 \\
\hline 24 & .0690 & .1114 & .1669 & .2079 \\
\hline 25 & .0663 & .1072 & .1608 & .2004 \\
\hline 26 & .0661 & .1033 & .1575 & .1936 \\
\hline 27 & .0615 & .0997 & .1500 & .1872 \\
\hline 28 & .0594 & .0962 & .1450 & .1811 \\
\hline 29 & .0574 & .0931 & .1404 & .1754 \\
\hline 30 & .0555 & .0901 & .1360 & .1698 \\
\hline
\end{tabular}

*One-tailed test.

\section{REFERENCES}

BAKAN, D. The test of significance in psychological research. Psychological Bulletin, 1966, 66, 423-437.

Bolles, R., \& Messick, S. Statistical utility in experimental inference. Psychological Report, 1958, 4, 223-227.

DWYER, J. H. Analysis of variance and the magnitude of effects: A general approach. Psychological Bulletin, 1974, 81, 731-737.

FlaGg, S. F., \& Medin, D. L. Constant irrelevant cues and stimulus generalization in monkeys. Journal of Comparative and Physiological Psychology, 1973, 85, 339-345.

Friedman, H. Magnitude of experimental effect and a table for its rapid estimation. Psychological Bulletin, 1968, 70, 245-251.

Gelfand, D. M., Hartman, D. P., Walder, P., \& Page, B. Who reports shoplifters? A field-experimental study. Journal of Personality and Social Psychology, 1973, 25, 276-285.

Gaito, J., \& Firth, J. Procedures for estimating magnitude of effects. The Journal of Psychology, 1973, 83, 151-161.

Hays, W. L. Statistics for the social sciences (2nd ed.). New York: Holt, Rinehart, and Winston, 1973.

LEvine, M. Scientific method and the adversary model: Some preliminary thoughts. American Psychologist, 1974, 29, 661-677.

Minturn, E. B. A proposal of significance. American Psychologist, 1971, 26, 669-670.

Skipper, J. K., Guenther, A. L., \& Nass, G. The sacredness of .05: A note concerning the use of statistical levels of significance in social science. The American Sociologist, 1967, 2, 16-18. 